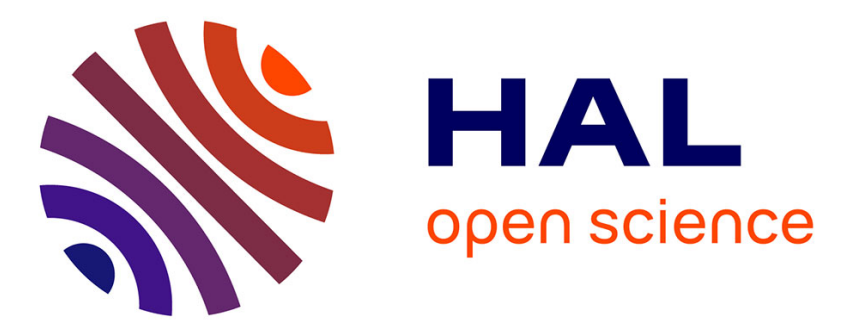

\title{
Virtual Proxemics: Locomotion in the Presence of Obstacles in Large Immersive Projection Environments
}

Ferran Argelaguet Sanz, Anne-Hélène Olivier, Gerd Bruder, Julien Pettré, Anatole Lécuyer

\section{- To cite this version:}

Ferran Argelaguet Sanz, Anne-Hélène Olivier, Gerd Bruder, Julien Pettré, Anatole Lécuyer. Virtual Proxemics: Locomotion in the Presence of Obstacles in Large Immersive Projection Environments. Proceedings of IEEE Virtual Reality Conference, Mar 2015, Arles, France. pp.75 - 80. hal-01149962

\author{
HAL Id: hal-01149962 \\ https://hal.inria.fr/hal-01149962
}

Submitted on 7 May 2015

HAL is a multi-disciplinary open access archive for the deposit and dissemination of scientific research documents, whether they are published or not. The documents may come from teaching and research institutions in France or abroad, or from public or private research centers.
L'archive ouverte pluridisciplinaire HAL, est destinée au dépôt et à la diffusion de documents scientifiques de niveau recherche, publiés ou non, émanant des établissements d'enseignement et de recherche français ou étrangers, des laboratoires publics ou privés. 


\section{Virtual Proxemics: Locomotion in the Presence of Obstacles in Large Immersive Projection Environments}

\author{
Fernando Argelaguet Sanz* \\ Inria
}

\author{
Anne-Hélène Olivier ${ }^{\dagger}$ \\ Inria \\ Anatole Lécuyer \\ Inria
}

\author{
Gerd Bruder \\ Universität Hamburg
}

Julien Pettré ${ }^{\S}$

Inria
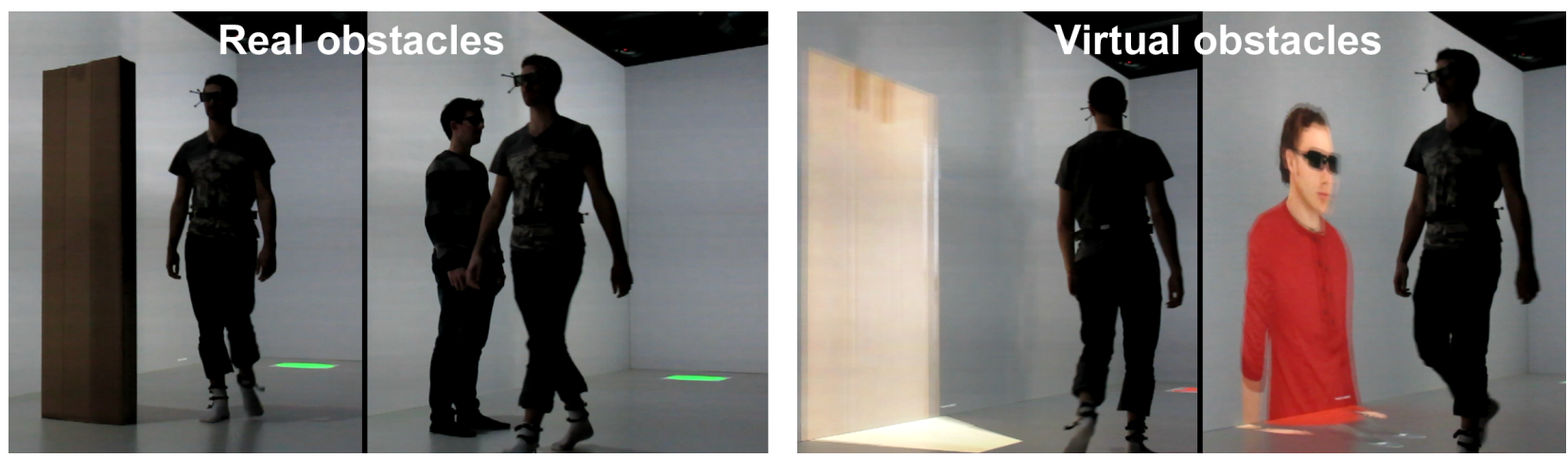

Figure 1: Experimental conditions considered on the collision avoidance experiment. We analyzed the participants' avoidance behavior for real (left) and virtual objects (right), considering anthropomorphic and inanimated objects.

\begin{abstract}
In this paper, we investigate obstacle avoidance behavior during real walking in a large immersive projection setup. We analyze the walking behavior of users when avoiding real and virtual static obstacles. In order to generalize our study, we consider both anthropomorphic and inanimate objects, each having his virtual and real counterpart. The results showed that users exhibit different locomotion behaviors in the presence of real and virtual obstacles, and in the presence of anthropomorphic and inanimate objects. Precisely, the results showed a decrease of walking speed as well as an increase of the clearance distance (i. e., the minimal distance between the walker and the obstacle) when facing virtual obstacles compared to real ones. Moreover, our results suggest that users act differently due to their perception of the obstacle: users keep more distance when the obstacle is anthropomorphic compared to an inanimate object and when the orientation of anthropomorphic obstacle is from the profile compared to a front position. We discuss implications on future large shared immersive projection spaces.
\end{abstract}

Index Terms: H.5.1 [Information Interfaces and Presentation]: Multimedia Information Systems-Artificial, Augmented, and Virtual Realities; I.3.7 [Computer Graphics]: Three-Dimensional Graphics and Realism-Virtual Reality

\footnotetext{
*e-mail: fernando.argelaguet_sanz@inria.fr

†e-mail: anne-helene.olivier@inria.fr@inria.fr

†e-mail:gerd.bruder@uni-hamburg.de

$\S$ e-mail:julien.pettre@inria.fr@inria.fr

Ile-mail: anatole.lecuyer@inria.fr@inria.fr
}

\section{INTRODUCTION}

CAVE-like immersive projection environments enable users to see both virtual and real objects, including the user's own body. With recent advances in VR technologies it becomes possible to build large-scale tracked immersive projection environments, which enable users to control their position in a large region of interest by real walking. Moreover, advances in stereoscopic display technologies [10] or light-field projection displays [2] may enable natural perspectives for multiple users in the near future. In such environments virtual and real objects as well as multiple users or avatars may coexist in the same interaction space. Hence, it becomes important to gain an understanding of how the user's behavior is affected by the differences in perception and affordances of such real and virtual obstacles. Particularly, in this paper we analyze potential changes in the user's locomotion behavior when avoiding virtual and real obstacles. Understanding and faithfully simulating natural locomotion dynamics between the user and the environment is of great interest to many virtual reality (VR) applications, including architectural review, training, and 3D entertainment.

In the real world we navigate with ease by walking in the presence of obstacles, we develop avoidance strategies and behaviors which govern the way we locomote in the proximity of physical objects and other persons during everyday tasks. Humans accurately perceive and control their self-motion - i.e., acceleration, speed and direction of travel, relative to their surroundings - based on consistent multi-sensory feedback including vestibular, proprioceptive, and efferent copy signals as well as visual information. However, realistic behavioral dynamics in immersive virtual environments (IVEs) are difficult to achieve especially due to the diminished perception of the virtual content [12]. Although modern real-time rendering systems can create compelling immersive experiences, offering most of the spatial visual cues we can find in the real world [17], not all visual cues can be faithfully reproduced when displaying virtual objects or humans. In this scope, it is wellknown that users tend to over- or underestimate spatial relations in 
immersive virtual environments [11]. Moreover, natural behavior while immersed is often limited by the user's illusions of place and plausibility [15].

This paper presents an experiment which analyzes the locomotion behavior of users' in a large immersive projection environment $(10 \mathrm{~m})$ when avoiding static obstacles. To our knowledge, this is the first experiment on interaction with real and virtual obstacles during locomotion tasks in a large CAVE-like immersive environment. Furthermore, in order to enable the cross comparison of the different situations that might arise in a mixed environment the experiment considers both real and virtual, anthropomorphic and inanimate obstacles (see Figure 1).

The paper is structured as follows. First, Section 2 summarizes the related work on collision avoidance in real and virtual environments. Section 3 describes the experiment in which we compared collision avoidance behavior during real walking in a ten meter shared IVE both with real and virtual objects, followed by Section 4 which describes the results. In Section 5 we discuss the results of the experiment. Finally, Section 6 concludes the paper.

\section{Related Work}

\subsection{Collision Avoidance in the Real World}

Proxemics is a fundamental concept when focusing on collision avoidance: it describes how humans manage space during interactions with their environment. Especially, a lot of work focused on the personal space that surrounds an individual. The personal space can be considered portable territory around an individual that others should not invade. It regulates the psychophysical distance that the individual needs to maintain in order to feel comfortable. According to Goffman [8], the personal space can be represented as an oval, narrow to the sides of the individual and long in front of him/her. In locomotion, personal space can be defined as a protective zone maintained around the walker that lets a sufficient delay to perceive unexpected events or obstacles and then allows to plan and perform required locomotor adjustments [16]. Gérin-Lajoie et al. [6] have experimentally confirmed Goffman's observations and found that this area can be defined by an elliptic curve.

A large amount of work has considered the way humans maintain this security distance by adapting their motion to avoid collisions with obstacles on their travel path. From a global point of view, the interactions between a walker and the environment can be modeled as a pair of coupled dynamical systems [4]. Heading is adapted according to the distance and the angle between the walker and stationary goals and obstacles: goals act as attractors and obstacles as repellors. Other authors suggested that path planning precedes dynamic control to navigate in a cluttered environment [14]. From a local point of view, some studies focused on the circumvention of non-human static obstacles [19]. Vallis and MacFadyen [19] showed that a walker avoids a cylinder ( $2 \mathrm{~m}$ high, $0.23 \mathrm{~m}$ diameter) using anticipatory locomotor adjustments three steps before the obstacle. Step width is adapted before obstacle crossing compared to a control situation in straight walking inducing a mediolateral center of mass displacement around $50 \mathrm{~cm}$ and the approaching velocity is constant. Other studies focused on the circumvention of human-shaped obstacles. Gérin-Lajoie et al. [6] demonstrated also anticipatory locomotor adjustments to avoid a mannequin on the travel path. Two phases in the avoidance where identified: an anticipatory phase with an initial deviation of the center of mass six steps before the obstacle and an adjustment of the trajectory during the last stride before the obstacle; and a clearance phase when the walker circumvents the obstacle. They showed that the avoidance strategy is initiated at a constant distance from the obstacle and that the clearance distance keeps constant values around one-third of the walkers' step-length.

\subsection{Collision Avoidance in Virtual Environments}

Since most CAVE-like systems do not provide sufficient interaction space to consider walking over more than a few meters, collision avoidance while walking in virtual environments has been studied only in head-mounted display (HMD) environments. Fink et al. [5] focused on collision avoidance of non-human stationary obstacles during goal reaching tasks. They compared tasks performed in physical and virtual environments. They showed some differences in the generated trajectories such as a larger lateral deviation, a larger obstacle clearance as well as a slower walking speed in virtual conditions compared to real ones. Gérin-Lajoie et al. [7] considered collision avoidance with a static cylinder. Performed in an HMD environment, they showed that the global shape of the personal space is preserved, but is slightly increased in virtual conditions compared to real ones. Moreover, Bailenson et al. [1] took interest in the interpersonal distance between a human and a virtual human in an HMD environment. They showed that participants maintained greater distance from virtual humans when walking towards them from their fronts compared to their backs. They also maintain a larger personal space to virtual agents who engaged them in mutual gaze.

In summary, all studies agree that humans keep a comparable behavior when avoiding virtual obstacles as they do with real ones. However, there are still not clear the causes of why humans consistently keep greater distances from virtual obstacles than from they real counterparts. While Fink et al. [5] hypothesize that the differences could be explained by the increased uncertainty about the obstacle egocentric location in IVEs, Gérin-Lajoie et al. [7] hypothesize that the differences could be due to limited field of view. HMDs have a limited field of view which restrict the user's view to the virtual environment (VE). For example, Fink. et al [5] used a HMD with a FoV of $60^{\circ} \mathrm{H}$ and Gérin-Lajoie et al. [7] an HDM with a FoV of $40^{\circ} \mathrm{H}$. In addition to the limited field of view, collision avoidance behavior is dominated by visual information from the VE, although extraretinal cues and fear to collide with obstacles in the real world often reduce natural locomotion behavior and gait [12].

\section{Behavioral Dynamics Experiment}

In this section we describe the experiment which we conducted to analyze differences in collision avoidance behavior of humans during real walking in the presence of real or virtual obstacles in a large shared IVE. The experimental task consisted in walking a distance of $8 \mathrm{~m}$ through the interaction space while avoiding a visible obstacle of either anthropomorphic or geometrical shape that was placed straight in front of the participants.

\subsection{Procedure}

Participants were instructed to walk from one side of the IVE to the opposite end at comfortable speed while avoiding any visible obsta-

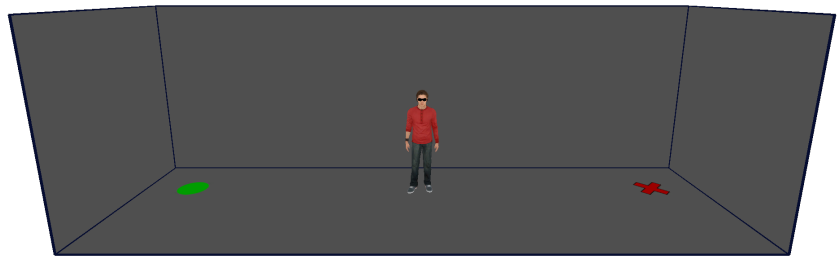

Figure 2: Layout of the experiment considering the physical boundaries of the IVE. The green dot represents the starting point and the red cross the end point of the path. The obstacle was placed in the middle of the working space, at $1 \mathrm{~m}$ from the front screen and at $2 \mathrm{~m}$ of the missing wall. 
cle. Additional visual feedback was provided to indicate the initial and final point of the trajectory via virtual markers projected on the floor (see Figure 2). During the collision avoidance task, only the obstacle was visible and the background was lighted with a medium gray color (see Figure 1). Participants had to walk a total trajectory of $8 \mathrm{~m}$ with the obstacle to avoid being placed in the middle. The center point of the obstacle was at $1 \mathrm{~m}$ of the front wall of the CAVE-like environment and at $2 \mathrm{~m}$ from the missing wall. The position of the virtual object was chosen to ensure that no additional physical constraints altered the locomotion behavior. In addition, this ensured that for all conditions participants always avoided the obstacle from the same side. Finally, it made sure that the virtual obstacles would be visible over the entire trajectory without being clipped by the boundary of the projection screens.

We tested a human and a simple geometrical object using each a real-world object and a virtual replica model of the physical counterpart (see Figure 3). Regarding the virtual human, we used a visually faithful textured 3D model of a lab member (male, $1.78 \mathrm{~m}$ height, $78 \mathrm{~kg}$ weight, $1.62 \mathrm{~m}$ eye height, $0.5 \mathrm{~m}$ shoulder width, $0.3 \mathrm{~m}$ depth). The virtual avatar was animated with an idle animation. The geometrical object was a cardboard box which matched the height, depth, and shoulder width of the human obstacle. The virtual cardboard was textured using pictures of the real one.

In order to focus participants on the tasks no communication between experimenter and participant was performed during the experiment after the initial training phase, in which we ensured that participants correctly understood the task. Written instructions were displayed on a computer prior to the experiment. Participants were allowed to take short breaks at any time between trials. For practical reasons, participants took short breaks each time there was the need to change the physical obstacles. The breaks were informed through a virtual panel appearing at the center of the IVE. The person playing the role of the real human obstacle had unnoticeable virtual guides for the participant in order to ensure that he was able to change his orientation correctly. Furthermore, he was wearing sun glasses to avoid eye-contact.

\subsection{Apparatus}

The experiment was conducted in a $9.6 \mathrm{~m} \times 3 \mathrm{~m} \times 3.1 \mathrm{~m}$ (width, depth, and height) 4-sided immersive projection setup equipped with 16 Barco Galaxy projectors at $15 \mathrm{MPixels}$ resolution in total. The missing wall was closed during the experiment using an opaque black lightshield. The participants wore shutter glasses (Volfoni ActivEyes Pro Radiofrequency for stereoscopic visual stimulus presentation) during all the experiment. The shutter glasses were tracked with 6 degrees of freedom (DOF) passive markers in the laboratory using an ART optical tracking system with 16 cameras at an update rate of $60 \mathrm{~Hz}$.

\subsection{Design}

We used a repeated-measures within-subjects design. The independent variables were the obstacle (cardboard box, human), the nature of the obstacle (real, virtual), the orientation of the obstacle $(\omega \in\{0,90,180,270\}$ degrees $)$ and the direction of the trajectory (left-to-right, right-to-left). Due to the symmetrical layout of the cardboard box we only considered 2 orientations of the obstacle, either facing the long or short side of the projection setup.

For the human obstacle participants completed 4 (orientation) $\times$ 2 (nature) $\times 2$ (direction) trials, as well as 2 (orientation) $\times 2$ (nature) $\times 2$ (direction) trials with the box-shaped obstacle. The order of the obstacle (real/virtual, cardboard box/human) was counterbalanced using a Latin-squared design, while the order of the orientation was randomized for each obstacle. For each combination, we performed four repetitions, resulting in 96 trials per participant. Additionally, at the beginning of the experiment, participants performed eight trials without any obstacle in order to gather baseline recordings.

Regarding the dependent variables, we recorded participants' trajectory as the horizontal position along time of their glasses tracked by the ART system. Of the entire $8 \mathrm{~m}$ walking trajectory we only considered the part from $x=-2.5 \mathrm{~m}$ until $x=2.5 \mathrm{~m}$ (the obstacle was at $x=0$ ) avoiding the accelerations and decelerations of the start and the end of the locomotion. The path information allowed us to compute the participants' average walking speed and speed profile. We also computed the maximal lateral deviation from a straight line between the starting and ending point of the trajectory as well as clearance defined as the minimal distance between the participant and the obstacle.

Furthermore, we collected demographic information with a questionnaire before the experiment and measured the participants' sense of presence with the Slater-Usoh-Steed (SUS) questionnaire [18], as well as simulator sickness with the Kennedy-Lane SSQ [9] before and after the experiment. The total time per participant including pre-questionnaires, instructions, training, experiments, breaks, and debriefing was 1 hour. Participants were immersed in the VE for about 45 minutes.

Considering our design, we hypothesize:

H1 Subjects will exhibit different locomotion trajectories with virtual obstacles vs. real obstacles. As found in previous experiments using HMDs, we expect slower walking speed and larger avoidance distance and clearance with virtual obstacles.

H2 Subjects will exhibit different locomotion trajectories during interaction with human vs. box obstacles. We expect that greater clearance is set with human obstacles, because social distances add to pure collision avoidance clearance.

H3 The orientation of the human obstacle will result in changes in the clearance distance, but at the opposite, the orientation of the box obstacle won't.

\subsection{Participants}

We recruited 17 participants for our experiment. 14 of them were male, 3 were female (ages $21-38, M=26.8$ ). The participants were either students or professionals in computer science or engineering. 14 participants were right-handed, 3 participants were lefthanded. The Lateral Preference Inventory questionnaire [3] confirmed that 14 participants were right-handed and 3 left-handed (rating scale $-4=$ left, $4=$ right, $\mathrm{M}=2.53, \mathrm{SD}=2.60$ ). Moreover, it showed that 15 participants were right-footed and 2 left-footed $(\mathrm{M}=2.29, \mathrm{SD}=2.17) .15$ participants were right-eyed and 1 lefteyed whereas the test was inconclusive for 1 participant $(M=2.71$, $\mathrm{SD}=2.11)$

8 participants wore glasses and 3 wore contact lenses during the experiment. One participant reported red-green color weakness; no other known vision disorders or displacements of balance were reported by the participants. Using the technique proposed by Willemsen et al. [20] we measured the interpupillary distance (IPD) of each participant before the experiment started $(\mathrm{M}=6.32 \mathrm{~cm}, \mathrm{SD}=0.28 \mathrm{~cm})$. Prior to the experiment we measured the eye height $(\mathrm{M}=1.65 \mathrm{~cm}, \mathrm{SD}=0.08 \mathrm{~cm})$ and the shoulder width $(\mathrm{M}=38.44 \mathrm{~cm}, \mathrm{SD}=3.84 \mathrm{~cm})$ of each participant. 16 participants reported previous experience with 3D stereoscopy (rating scale $0=$ yes, $4=$ no, $\mathrm{M}=1.47, \mathrm{SD}=1.23$ ). 15 participants had participated in a study in the immersive projection setup before.

\section{Results}

Statistics were performed using the Statistica Software. Normality was assessed using a Kolmogorov-Smirnov test. To evaluate the influence of the experimental factors on average walking speed, maximal lateral deviation, and clearance, we performed separate threeway analysis of variance (ANOVA) with repeated measures on the 


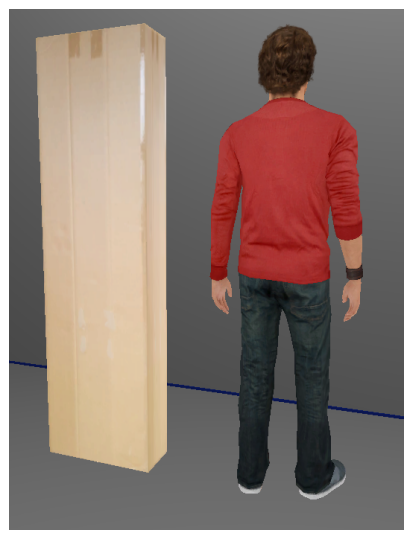

(a) $0^{\circ}$

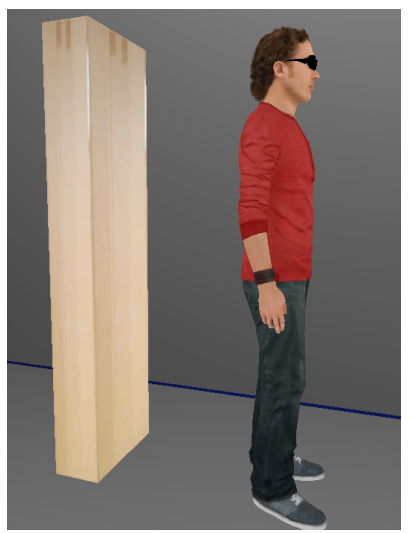

(b) $90^{\circ}$
Figure 3: Orientation configuration for the obstacles when users walked from the left to the right. The orientation of the human obstacle for the $180^{\circ}$ and $270^{\circ}$ follow a clockwise rotation. For right to left conditions, $90^{\circ}$ and $270^{\circ}$ configurations are shifted.

conditions (2: real,virtual), the obstacle (2: human and box) and the orientation of the obstacle $\left(2: 0^{\circ}\right.$ and $\left.90^{\circ}\right)$. When appropriate, Bonferroni post-hoc tests were used to further analyze significant effects. To consider all the orientations, we also performed for the human obstacle condition a two-way analysis of variance (ANOVA) with repeated measures on the conditions (2: real,virtual) and the orientation of the obstacle $\left(4: 0^{\circ}, 90^{\circ}, 180^{\circ}\right.$ and $\left.270^{\circ}\right)$ associated with Bonferroni post-hoc tests.

Average values of the kinematic variables depending on the experimental conditions are reported in Figure 4. Figure 5 shows the average trajectories performed by participants to avoid the obstacle depending on the orientation of the obstacle $(a, b)$. We excluded the few data sets from the analysis in which the participant walked between the front screen and the obstacle (5\% trials). Furthermore, no ordering effects were observed.

Walking Speed Results showed that only the real/virtual condition influences the average walking speed $(F(1,16)=4.78$, $\left.p<0.05, \eta_{p}^{2}=0.23\right)$ : walking speed is significantly higher in real conditions $(1.17 \mathrm{~m} / \mathrm{s})$ than in virtual conditions $(1.13 \mathrm{~m} / \mathrm{s})$.

Maximal Lateral Deviation Results showed an influence of $\mathrm{real} / \mathrm{virtual}$ conditions $\left(F(1,16)=16.03, p<0.005, \eta_{p}^{2}=0.50\right)$, the obstacle $\left(F(1,16)=17.58, p<0.001, \eta_{p}^{2}=0.52\right)$ and the orientation $\left(F(1,16)=103.05, p<0.001, \eta_{p}^{2}=0.86\right)$. Bonferroni post-hoc tests showed that maximal deviation is significantly higher in virtual conditions $(0.71 \mathrm{~m})$ compared to real ones $(0.66 \mathrm{~m})$; it is higher when the obstacle is a human $(0.72 \mathrm{~m})$ compared to a box $(0.65 \mathrm{~m})$; and it is higher when orientation is $90^{\circ}(0.72 \mathrm{~m})$ compared to $0^{\circ}(0.65 \mathrm{~m})$.

When considering the human obstacle condition only, results showed that real/virtual condition $(F(1,15)=8.08, \quad p<0.01$, $\left.\eta_{p}^{2}=0.37\right), \quad$ orientation $\left(F(3,45)=6.04, \quad p<0.005, \quad \eta_{p}^{2}=0.29\right)$ as well as the interaction between real/virtual and orientation $\left(F(3,45)=11.35, \quad p<0.001, \quad \eta_{p}^{2}=0.43\right)$ influence the maximal deviation. Bonferroni post-hoc test showed that maximal deviation is higher in virtual conditions $(0.76 \mathrm{~m})$ compared to real conditions $(0.69 \mathrm{~m})$. Maximal lateral deviation is also higher for $90^{\circ}$ and $270^{\circ}(0.74 \mathrm{~m})$ than for $0^{\circ}(0.70 \mathrm{~m})$. Considering the interaction effect between real/virtual conditions and orientation of the human obstacle, post-hoc tests showed that maximal lateral deviation is higher in virtual conditions for orientations $90^{\circ}, 180^{\circ}$ and $270^{\circ}$.
Clearance Distance Results showed an influence of the real/virtual conditions $\left(F(1,16)=7.51, p<0.05, \eta_{p}^{2}=0.32\right)$, the obstacle $\left(F(1,16)=19.16, p<0.001, \eta_{p}^{2}=0.54\right)$ and the orientation $\left(F(1,16)=61.91, p<0.001, \eta_{p}^{2}=0.79\right)$. Bonferroni post-hoc tests showed that clearance distance is higher in virtual conditions $(0.61 \mathrm{~m})$ compared to real conditions $(0.56 \mathrm{~m})$; it is higher when the obstacle is a human $(0.62 \mathrm{~m})$ compared to a box $(0.55 \mathrm{~m})$; and it is higher when orientation is $0^{\circ}(0.61 \mathrm{~m})$ compared to $90^{\circ}(0.55 \mathrm{~m})$.

For the human obstacle condition only, ANOVA showed that $\mathrm{real} / \mathrm{virtual}$ condition $\left(F(1,15)=7.06, \quad p<0.05, \eta_{p}^{2}=0.32\right)$, orientation $\left(F(3,45)=38.08, \quad p<0.001, \quad \eta_{p}^{2}=0.71\right)$ as well as the interaction between real virtual conditions and orientation $\left(F(3,45)=16.23, p<0.001, \eta_{p}^{2}=0.52\right)$ significantly influenced the clearance distance. Bonferroni post-hoc test show that clearance distance is higher in virtual conditions $(0.66 \mathrm{~m})$ compared to real conditions $(0.60 \mathrm{~m})$ and that it is higher when orientation is $0^{\circ}(0.66 \mathrm{~m})$ or $180^{\circ}(0.70 \mathrm{~m})$ compared to $90^{\circ}(0.58 \mathrm{~m})$ and $270^{\circ}(0.57 \mathrm{~m})$. Furthermore, the virtual human condition at $180^{\circ}$ leads to significantly higher clearance.

\section{Questionnaires}

We measured a mean SSQ-score of $\mathrm{M}=18.118$ ( $\mathrm{SD}=2.261)$ before the experiment, and a mean score of $\mathrm{M}=18.647(\mathrm{SD}=2.370)$ after the experiment. There was no significant difference of the SSQscore before and after the experiment $(t(16)=1.21, p=0.245)$. The mean SUS-score for the reported sense of feeling present in the VE was $\mathrm{M}=4.30(\mathrm{SD}=0.60)$, which indicates a reasonably high level of presence. Participants judged whether they were more careful avoiding the real than the virtual box (rating scale $0=$ yes, $4=$ no, $\mathrm{M}=3.35, \mathrm{SD}=1.22$ ) as well as the real than the virtual human (rating scale $0=$ yes, $4=$ no, $\mathrm{M}=2.77, \mathrm{SD}=1.48$ ). Additionally, participants judged whether they had the impression of being able to walk "through" a virtual obstacle (rating scale $0=$ yes, $4=$ no, $M=2.29$, $\mathrm{SD}=1.26)$.

\section{Discussion}

When comparing the virtual and real conditions, we observe that our results show the same tendency as previous studies done in HMDs [5, 7]. Walking speed is slower, and the maximal lateral deviation as well as the clearance are higher when avoiding virtual obstacles than real ones, thus supporting H1. This results are also observed in the subjective questionnaires were users judged in general to be more careful when avoiding virtual objects. Although, it is not possible to perform a quantitative comparison between our study and previous ones due to differences in the experimental protocols, we obtained lower differences among virtual and real conditions. For example, in Fink et al. [5] mean speed differences where $\Delta=0.13 \mathrm{~m} / \mathrm{s}$ while in our experiment where $\Delta=0.04 \mathrm{~m} / \mathrm{s}$. But, do large IVEs or mixed reality environment induce less bias in kinematic data than HMDs? Additional work and dedicated experiments are required to answer this question. Nevertheless, large IVEs enable more ecological VR situations (no helmet, no limitation on field of view). Considering the absence of direct body feedback during locomotion with HMDs as well as the potential impact of hard- and software factors in HMD setups, future work may focus on identifying the contributing factors for this difference.

Regarding the nature of the obstacle (human vs. box), the maximal lateral deviation of the path and the clearance distance are higher for anthropomorphic obstacles whereas the walking speed is not affected. Participants keep greater distances when the obstacle is anthropomorphic in comparison with an inanimate obstacle. This effect suggests that participants take greater security distance to avoid the collision. This result also supports $\mathrm{H} 2$.

Considering the orientation, results showed an influence on the maximal lateral deviation of the locomotor path with higher values 

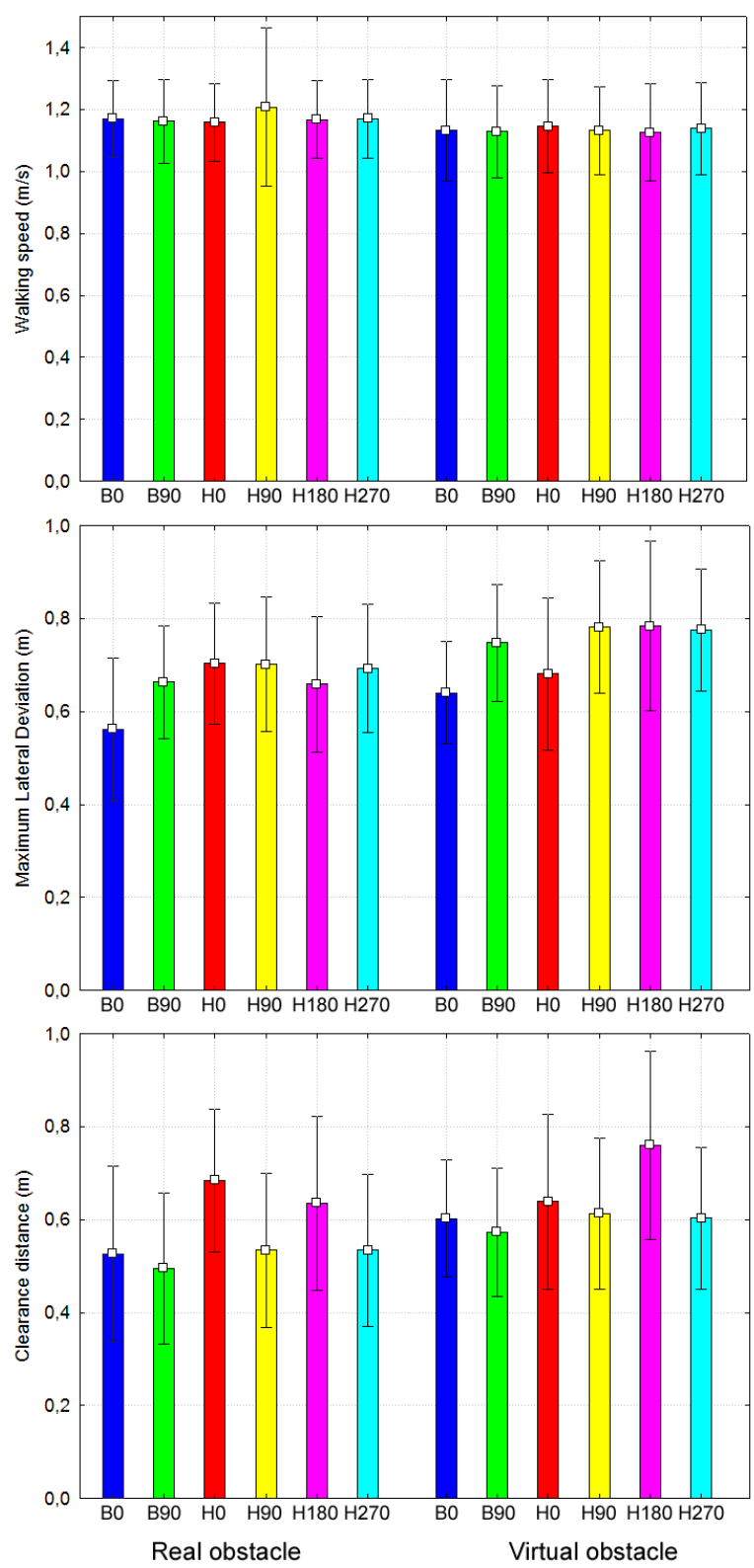

Figure 4: Bar plots reporting the mean values $\pm S D$ of speed, maximum lateral deviation and clearance distance computed for all experimental conditions ( $\mathrm{B} 0=\mathrm{Box}$ obstacle with an orientation of $0^{\circ}$ $\mathrm{B} 90=\mathrm{Box}$ obstacle with an orientation of $90^{\circ}, \mathrm{HO}=$ Human obstacle with an orientation of $0^{\circ}, \mathrm{H} 90=$ Human obstacle with an orientation of $90^{\circ}, \mathrm{H} 180=$ Human obstacle with an orientation of $180^{\circ}$, $\mathrm{H} 270=$ Human obstacle with an orientation of $270^{\circ}$ ).

for $90^{\circ}$ and $270^{\circ}$ conditions. This effect can easily be explained by the experimental conditions since obstacles at $90^{\circ}$ and $270^{\circ}$ obstruct more the path in front of the walker and require more lateral adaptations to avoid the collision. More interesting is the influence of the orientation on the clearance distance when the obstacle is anthropomorphic. Orientations of the obstacles at $0^{\circ}$ and $180^{\circ}$ resulted in significantly larger clearance distances, thus supporting H3. This effect of orientation on the clearance distance may be explained by the shape of the personal space, that was shown to be elliptic [6] with larger distance in the anteroposterior axis than the mediolateral one. In that sense, other authors attribute some role

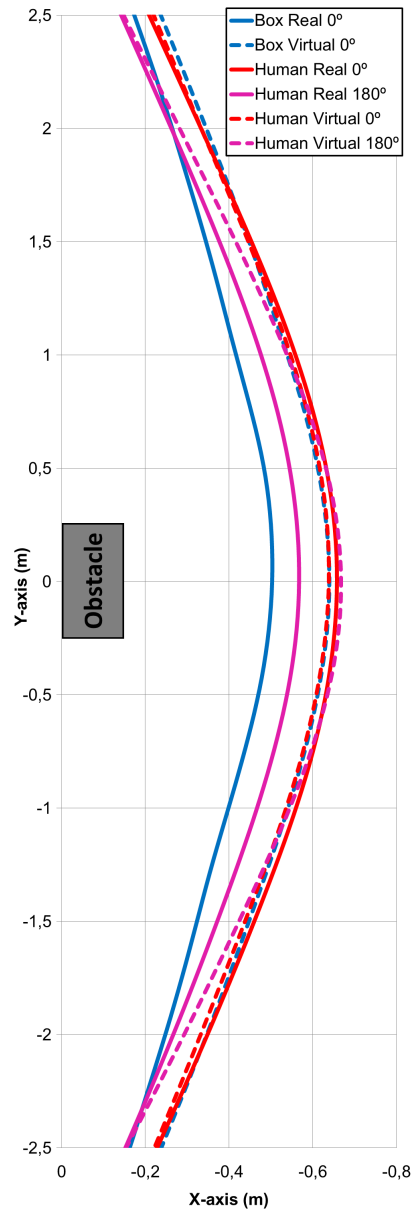

(a) Orientations $0^{\circ}$ and $180^{\circ}$

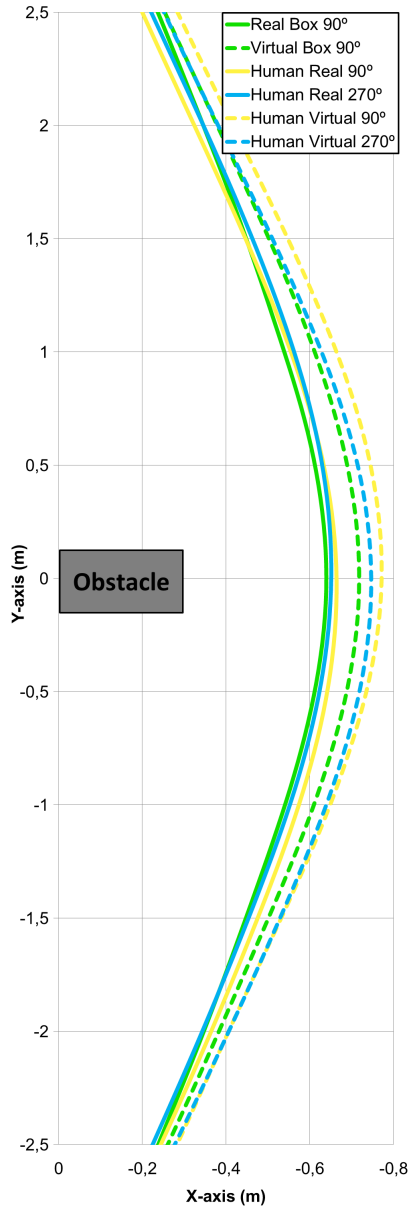

(b) Orientations $90^{\circ}$ and $270^{\circ}$
Figure 5: Average trajectories performed by participants to reach a target while avoiding a static obstacle in their travel path. Walking direction goes from negative $y$-values to positive $y$-values.

dependent strategies for collision avoidance between two walkers to the asymmetry of personal space [13]. This suggests that even in virtual conditions, users consider the personal space of the human obstacle in the collision avoidance task. When the human obstacle is viewed from profile, the larger part of the personal space is in the direction of the mediolateral adaptation, which should induce larger clearance distances as illustrated in Figure 6. However, we did not observe a difference between front and back orientation of the virtual human such as observed by Bailenson et al. [1] who showed that a user lets more distance with a virtual human when approaching it from the front than from the back. This difference may be explained by the difference in the experimental protocol. In their study, the goal was to approach the virtual human and not avoid it to go toward a target. Also, when users were instructed to approach the virtual human from the back, they first go towards the front and walk around it.

\section{Conclusion AND Future Work}

In this paper, we investigated obstacle avoidance behavior during real walking in IVEs. Contrary to previous studies, we considered a large immersive projection setup instead of an HMD. We compared in a kinematic analysis study how users avoid static obstacles of different types. We focused on real and virtual obsta- 


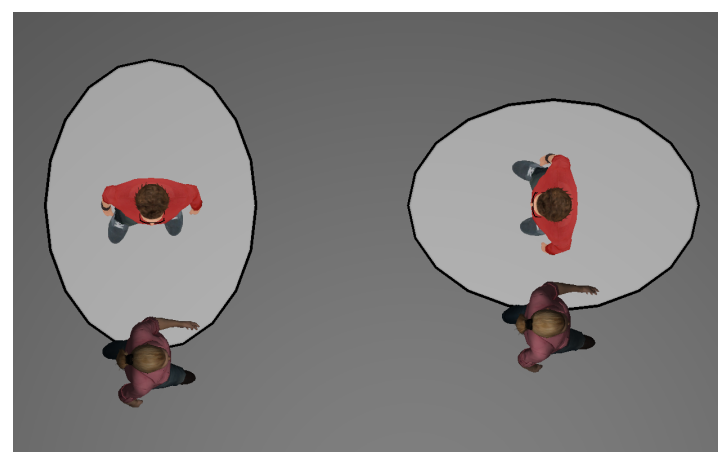

Figure 6: Hypothesis with regard of the influence of the personal space of the virtual character on the clearance distance let by the user in the collision avoidance task. The elliptical shape of the personal space results in changes on the clearance distance according to the orientation of the obstacle.

cles, which can take two forms, anthropomorphic or inanimate, and with different orientations (front, back and profile). First of all, the results showed changes on the collision avoidance behavior between virtual and real obstacles, which validates the findings of previous experiment for CAVE-like setups. However, although we observed differences in collision avoidance behavior between real and virtual obstacles, which indicate biases of natural locomotion introduced by the setup, their magnitude seem lower compared to typical results found in HMD environments. In addition, the differences between the real and virtual conditions, even being significant, showed small effects. This suggests that although the user's behavior in mixed environments varies depending on the nature of the stimulus, in our particular setup (and potentially in most projection-based systems), the user's locomotion behavior and the management of his/her interaction space is comparable with the ones in real life.

Considering these findings, our results open promising vistas for using large CAVE-like setups for socio-physical experiments, in particular in the fields of locomotion and behavioral dynamics. In reality it is difficult to obtain standard conditions to study proxemics because they depend on relative kinematics states (relative position and motion). In contrast to real conditions, VR opens the ability to accurately steer the virtual character with respect to the real subject motion. Future VR-based proxemics studies could consider the presence of moving obstacles and characters, different types of interactions between obstacles or characters and real subjects (e.g. reaching, meeting, fleeing), as well as multiple interactions. Some kind of interactions could require more detailed kinematics analysis, with full body motion capture data (e.g. feet placement, postures, swaying amplitudes).

\section{ACKNOWLEDGEMENTS}

This work was supported by the Vision Advanced Infrastructure for Research (VISIONAIR) project. Authors of this work are supported by the French National Research Agency ANR, project PERCOLATION number ANR-13-JS02-0008 and by the German Research Foundation.

\section{References}

[1] J. N. Bailenson, J. Blascovich, A. C. Beall, and J. M. Loomis. Interpersonal distance in immersive virtual environments. Personality \& social psychology bulletin, 29(7):819-33, 2003.
[2] T. Balogh, P. T. Kovacs, and A. Barsi. Holovizio 3D display system. In Proceedings of IEEE 3DTV Conference, pages 1-4, 2007.

[3] S. Coren. The lateral preference inventory for measurement of handedness, footedness, eyedness, and earedness: norms for young adults. Bulletin of the Psychonomic Society, 31(1):1-3, 1993.

[4] B. R. Fajen and W. H. Warren. Behavioral dynamics of steering, obstable avoidance, and route selection. Journal of Experimental Psychology: Human Perception and Performance, 29(2):343-362, 2003.

[5] P. W. Fink, P. S. Foo, and W. H. Warren. Obstacle avoidance during walking in real and virtual environments. ACM Transactions on Applied Perception (TAP), 4(1):2, 2007.

[6] M. Gérin-Lajoie, C. Richards, and B. McFadyen. The negociation of stationary and moving obstructions during walking: anticipatorylocomotor adaptations and preservation of personal space. Motor Control, 9:242-269, 2005

[7] M. Gérin-Lajoie, C. L. Richards, J. Fung, and B. J. McFadyen. Characteristics of personal space during obstacle circumvention in physical and virtual environments. Gait \& posture, 27(2):239-247, 2008.

[8] E. Goffman. Relations in public: microstudies of the public order. Basic Books, 1971.

[9] R. S. Kennedy, N. E. Lane, K. S. Berbaum, and M. G. Lilienthal. Simulator sickness questionnaire: An enhanced method for quantifying simulator sickness. International Journal of Aviation Psychology, 3(3):203-220, 1993.

[10] A. Kulik, A. Kunert, S. Beck, R. Reichel, R. Blach, A. Zink, and B. Froehlich. C1x6: A stereoscopic six-user display for co-located collaboration in shared virtual environments. ACM Transactions on Graphics, 30(6):188:1-188:12, 2011.

[11] J. M. Loomis and J. M. Knapp. Visual perception of egocentric distance in real and virtual environments. In H. L. J. Mahwah, N. J and M. W. Haas, editors, Virtual and adaptive environments, volume Virtual and adaptive environments, pages 21-46. 2003

[12] B. J. Mohler, W. B. Thompson, S. H. Creem-Regehr, H. L. Pick, and W. H. Warren. Visual flow influences gait transition speed and preferred walking speed. Experimental brain research, 181(2):221-8, 2007.

[13] A.-H. Olivier, A. Marin, A. Crétual, A. Berthoz, and J. Pettré. Collision avoidance between two walkers: Role-dependent strategies. Gait \& Posture, 38(4):751 - 756, 2013

[14] A. E. Patla, S. S. Tomescu, and M. G. Ishac. What visual information is used for navigation around obstacles in a cluttered environment? Canadian journal of physiology and pharmacology, 82(8-9):682-692, 2004.

[15] M. Slater. Place illusion and plausibility can lead to realistic behaviour in immersive virtual environments. Philosophical transactions of the Royal Society of London. Series B, Biological sciences, 364(1535):3549-57, 2009

[16] J. Templer. Human territoriality and space needs on stairs. In The Staircase: Studies of Hazards, Falls, and Safer Design. pp 61-70. MIT Press, 1992.

[17] W. Thompson, R. Fleming, S. Creem-Regehr, and J. K. Stefanucci. Visual Perception from a Computer Graphics Perspective. A. K. Peters / CRC Press, 2011.

[18] M. Usoh, E. Catena, S. Arman, and M. Slater. Using presence questionnaires in reality. Presence: Teleoperators in Virtual Environments, 9(5):497-503, 1999.

[19] L. A. Vallis and B. J. McFadyen. Locomotor adjustments for circumvention of an obstacle in the travel path. Experimental brain research, 152(3):409-414, 2003.

[20] P. Willemsen, A. A. Gooch, W. B. Thompson, and S. H. CreemRegehr. Effects of stereo viewing conditions on distance perception in virtual environments. Presence: Teleoperators and Virtual Environments, 17(1):91-101, 2008. 